\title{
Calculation of weak matrix elements in domain-wall QCD with the DBW2 gauge action
}

\author{
J. Noaki ${ }^{\mathrm{a}}$ for the RBC Collaboration* \\ ${ }^{\text {a}}$ RIKEN-BNL Research Center, Brookhaven National Laboratory, Upton, NY 11973-5000, USA
}

\begin{abstract}
We report the details of our ongoing quenched calculations of weak matrix elements using the combination of domain-wall fermions and the DBW2 gauge action on lattices with $a^{-1} \approx 3 \mathrm{GeV}$. A strategy to avoid the problem of fixed topological charge is introduced in generating gauge configurations. After studying the basic run parameters and elemental quantities, we present a preliminary result for the kaon B-parameter $\left(B_{K}\right)$.
\end{abstract}

\section{Introduction}

The combination of domain-wall fermions and renormalization group (RG) improved gauge actions has been proven to yield fermions with good chiral behavior, allowing progress in the calculation of weak matrix elements (WME) on the lattice [2]. On the other hand, two examples of open issues are the scaling behavior of $B_{K}$ and the effect of the charm quark which contributes to $K \rightarrow \pi \pi$ matrix elements through the quark loop contraction [3]. Both of these require the calculation of WME, using fermions with good chiral behavior, on finer lattices than have been employed so far.

In this article, we present the current results from an ongoing quenched numerical simulation by the RBC Collaboration. It uses domainwall fermions (DWF) and the DBW2 gauge action at a scale $a^{-1} \approx 3 \mathrm{GeV}$ with physical spatial size $\approx 1.6 \mathrm{fm}$. Though the use of the RG-improved DBW2 gauge action improves chiral symmetry, it has been recognized that the topological charge of DBW2 gauge configurations generated by standard Monte Carlo techniques evolves very slowly [4. This fact motivated us to implement a strategy to generate gauge configurations whose topological charge is well-

\footnotetext{
${ }^{*}$ Current members of the collaboration are: Y. Aoki, T. Blum, N. Christ, M. Creutz, C. Dawson, T. Izubuchi, L. Levkova, X. Liao, G. Liu, R. Mawhinney, Y. Nemoto, J. Noaki, S. Ohta, K. Orginos, S. Prelovsek, S. Sasaki and A. Soni.
}

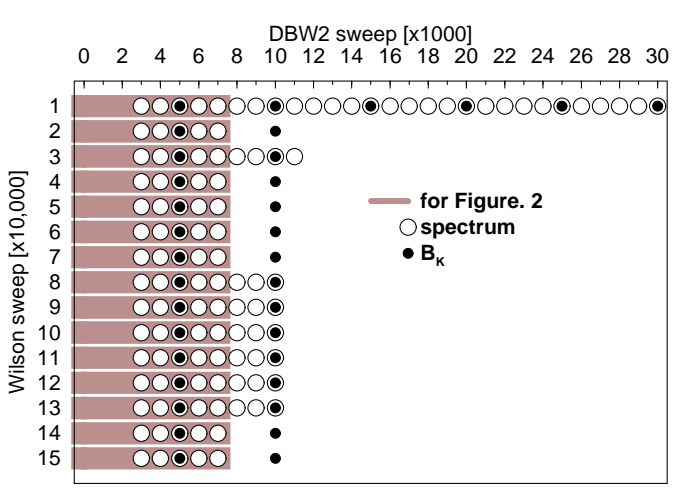

Figure 1. The gauge configurations used for spectrum (open circle) and $B_{K}$ (filled circle). Shadowed bars indicate data employed in Figure 2 .

distributed [1].

In the following, we discuss the details of our numerical simulation and present preliminary results for the meson spectrum, residual chiral symmetry breaking mass $m_{\text {res }}$ and $B_{K}$.

\section{Gauge configuration generation}

On a $24^{3} \times 48$ lattice, we generate gauge configurations using the Wilson action with $\beta=6.25$ in steps of 10,000 sweeps and use each of these as the initial configuration for a DBW2 evolution with $\beta=1.22$ in steps of 1000 sweeps. The status of our numerical simulation for 15 independent 
Table 1

Simulation parameters for observables.

\begin{tabular}{c|c|c}
\hline & spectrum & $B_{K}$ \\
\hline \hline size & \multicolumn{2}{|c}{$24^{3} \times 48$} \\
\hline$M_{5}$ & 1.7 & 1.65 \\
\hline$L_{s}$ & 8 & 10 \\
\hline$m_{f} a$ & $0.02,0.03,0.04$ & $\begin{array}{c}0.008-0.040 \\
\text { in step of } 0.008\end{array}$ \\
\hline \#sweep & every 1000 & every 5000 \\
\hline \#config. & 120 & 30 \\
\hline
\end{tabular}

DBW2 series is illustrated in Figure 1 .

The initial Wilson configurations have $a^{-1} \approx 3$ $\mathrm{GeV}$, the same lattice spacing desired for the DBW2 ensemble. The part of the topological charge produced by physical, long distance properties of the Wilson lattices should be very close to that of a fully sampled DBW2 ensemble, since at these weak couplings such a physical quantity is independent of the UV details of the action. We rely on this physical argument to allow us to use the topological distribution generated by the Wilson action as a starting point for our DBW2 ensemble. With the 15 Wilson configurations discussed here, we find $\left\langle Q_{\text {top }}\right\rangle=-0.60 \pm 3.11$. (For 50 configurations, we have $\left\langle Q_{\text {top }}\right\rangle=-0.32 \pm 3.36$, with $-10 \leq Q_{\mathrm{top}} \leq+7$.) For a few of the DBW2 evolutions, $Q_{\text {top }}$ changed by one unit, while for the longest 30,000 sweep evolution, $Q_{\text {top }}$ did not change. Thus, for the DBW2 action, we conclude that over-relaxed and heat-bath steps would not generate a reasonable sampling of topologies at these weak couplings.

\section{Hadron spectrum}

Simulation parameters for the spectrum calculation are summarized in the middle column of

Table 2

Spectrum results for $M_{5}=1.7, L_{s}=8$.

\begin{tabular}{cc}
\hline$m_{P S} / m_{V}$ & $0.612(11), 0.698(9), 0.756(7)$ \\
$a^{-1}$ & $2.89(12) \mathrm{GeV}$ \\
$m_{s} a / 2$ & $0.0157(11)$ \\
$m_{\mathrm{res}}$ & $1.011(35) \mathrm{MeV} \quad\left(M_{5}=1.7, L_{s}=8\right)$ \\
& $0.276(10) \mathrm{MeV} \quad\left(M_{5}=1.65, L_{s}=10\right)$ \\
\hline
\end{tabular}

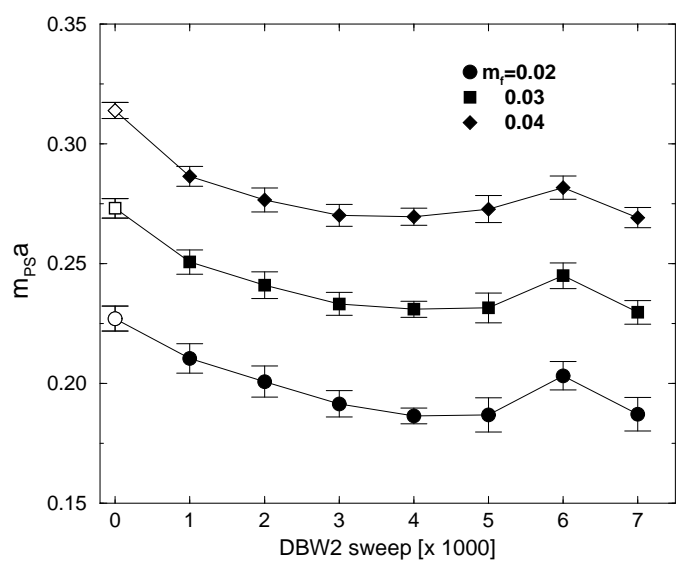

Figure 2. $m_{P S} a$ versus evolution in the DBW2 direction (filled symbols) obtained from averaging over 15 configurations in the vertical direction shown in Figure 1. Open symbols represent the data from the initial Wilson configurations.

Table 1. To investigate how our strategy works and determine optimal parameters for the calculation of WME, we studied meson masses and the residual quark mass $m_{\text {res }}$ which is induced by the explicit chiral symmetry breaking due to finite $L_{s}$.

It is important to estimate the equilibration time for the DBW2 evolutions, starting from the initial Wilson configurations. Figure 2 shows the results for the pseudoscalar meson mass $m_{P S} a$ for each $m_{f} a$ and averaged over the configurations indicated in Figure 1. In this figure, one observes that about 3000 sweeps are necessary for thermalization, which is consistent with a similar analysis for $m_{\text {res }}$. Our hadron spectrum determination only uses data from 3000 sweeps on.

Table 2 contains the results from our spectrum calculation with $M_{5}=1.7$ and $L_{s}=8$. The lattice scale $a^{-1}$ was determined from the rho meson mass at the chiral limit. We obtained the strange quark mass from the relation $m_{P S}^{2}=2 B_{0} m_{f}$. Further study of $m_{\text {res }}$ as a function of domainwall height $M_{5}$, gave 1.65 as the optimal value. Using this, we found $m_{\text {res }}<0.3 \mathrm{MeV}$ for the DBW2 action with $L_{s}=10$. This value for $L_{s}$ is used in the calculation of WME. 


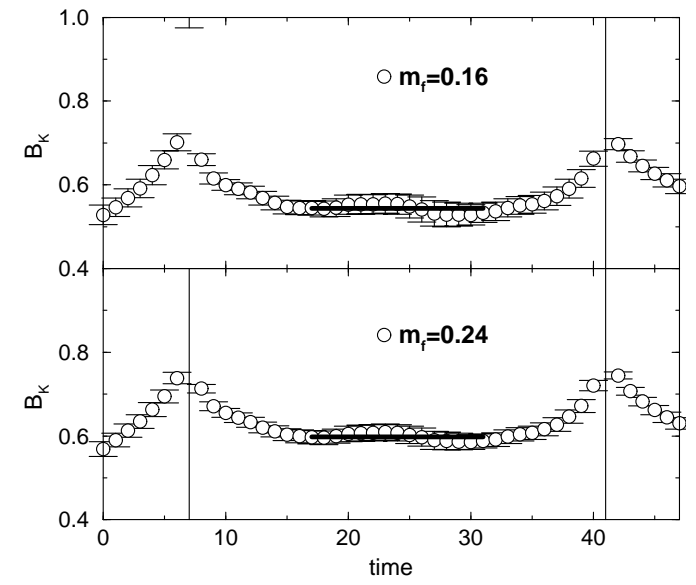

Figure 3. time dependence of $B_{K}$ for $m_{f} a=$ 0.016 (upper) and 0.024 (lower). The solid lines indicate constant fits.

\section{Kaon B parameter}

$B_{K}=\frac{\left\langle\bar{K}^{0}\left|\bar{s} \gamma_{\mu}\left(1-\gamma_{5}\right) d \bar{s} \gamma_{\mu}\left(1-\gamma_{5}\right) d\right| K^{0}\right\rangle}{\frac{8}{3}\left\langle\bar{K}^{0}\left|\bar{s} \gamma_{4} \gamma_{5} d\right| 0\right\rangle\left\langle 0\left|\bar{s} \gamma_{4} \gamma_{5} d\right| K^{0}\right\rangle}$

was calculated with the parameters summarized in the right column of Table 1. Quark propagators from Coulomb gauge fixed spatial wall sources located at $t=7$ and 41 were used in our calculation and each propagator is an average of ones with periodic and anti-periodic boundary conditions in the time direction.

One finds reasonable plateaus in $t$ for $B_{K}$ as shown in Figure 3. Figure 1 shows the unrenormalized value of $B_{K}$ as a function of $m_{f} a$ and a fit to the function $B_{K}\left(m_{f} a\right)=\xi_{0}[1+$ $\left.C m_{f} a \ln \left(m_{f} a\right)\right]+\xi_{1} m_{f} a$, where the value of $C$ is taken from chiral perturbation theory [5,6]. The data are well fit to this function. An uncorrelated fit has $\chi^{2} /$ dof $=0.14$. The interpolated value $B_{K}=0.539(21)$ at the physical point $m_{f} a=m_{s} a / 2$ is indicated by a filled square. We have not yet calculated the non-perturbative renormalization factor for $B_{K}$. Employing the perturbative one [7], the value of $B_{K}$ in the $\overline{\mathrm{MS}}$ NDR scheme can be estimated.

Previous works calculated $B_{K}(\mu=2 \mathrm{GeV})$ with DWF in similar physical spatial volumes 8 ,

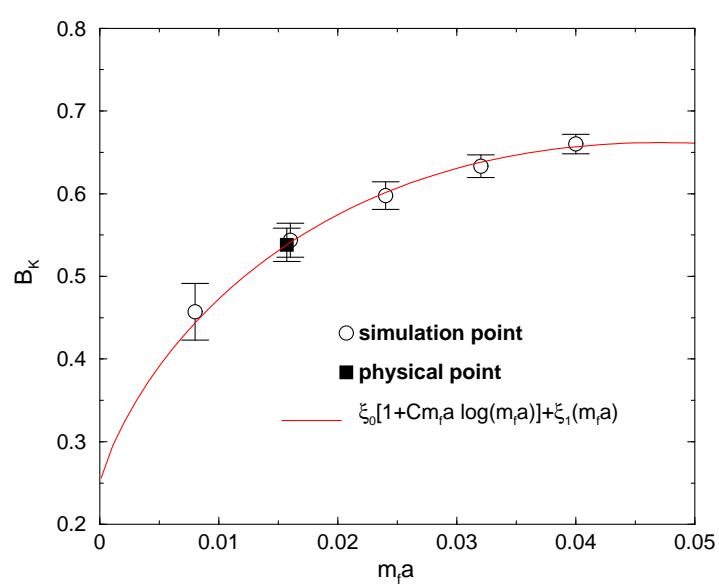

Figure 4. Lattice value of $B_{K}$ as a function of $m_{f} a$. The interpolated point (filled symbol) indicates the value at $m_{f} a=m_{s} a / 2$. The solid line denotes a fit that is described in the text.

9. While our preliminary result is consistent with both of these within roughly one and two standard deviations, respectively, it is smaller than results from larger physical volume [8].

We thank RIKEN, BNL and the U.S. DOE for providing the facilities essential for the completion of this work.

\section{REFERENCES}

1. We changed a part of data presented in the talk to correct a simple human error made in the random numbers used in generating the gauge configurations.

2. Review by N. Ishizuka, these proceedings.

3. See R. Mawhinney, in these proceedings.

4. K. Orginos et. al. (RBC Collaboration), Nucl. Phys. B (Proc. Suppl.) 106 (2002) 721.

5. S. Sharpe, Phys. Rev. D46 (1992), 3146.

6. M. Golterman and E. Pallante, JHEP 0008 (2000) 023.

7. S. Aoki et. al., hep-lat/0206013.

8. A. Ali Kahn et. al. (CP-PACS Collaboration), Phys. Rev. D64 (2001), 114506.

9. T. Blum et. al. (RBC Collaboration), hep-lat/0110075. 